Research Article

\title{
A New Method of Matrix Decomposition to Get the Determinants and Inverses of $r$-Circulant Matrices with Fibonacci and Lucas Numbers
}

\author{
Jiangming Ma $\mathbb{D}^{1},{ }^{1}$ Tao Qiu $\mathbb{D D}^{2}$ and Chengyuan $\mathrm{He} \mathbb{D}^{3}$ \\ ${ }^{1}$ School of Economics, Xihua University, Chengdu, Sichuan 610039, China \\ ${ }^{2}$ Sichuan Deyang No. 5 Middle School, Deyang, Sichuan 618000, China \\ ${ }^{3}$ School of Science, Xihua University, Chengdu, Sichuan 610039, China \\ Correspondence should be addressed to Chengyuan He; chengyuanh@163.com
}

Received 18 May 2021; Revised 14 October 2021; Accepted 29 October 2021; Published 18 November 2021

Academic Editor: Firdous A. Shah

Copyright (C) 2021 Jiangming Ma et al. This is an open access article distributed under the Creative Commons Attribution License, which permits unrestricted use, distribution, and reproduction in any medium, provided the original work is properly cited.

We use a new method of matrix decomposition for $r$-circulant matrix to get the determinants of $A_{n}=\operatorname{Circ}_{r}\left(F_{1}, F_{2}, \ldots, F_{n}\right)$ and $B_{n}=\operatorname{Circ}_{r}\left(L_{1}, L_{2}, \ldots, L_{n}\right)$, where $F_{n}$ is the Fibonacci numbers and $L_{n}$ is the Lucas numbers. Based on these determinants and the nonsingular conditions, inverse matrices are derived. The expressions of the determinants and inverse matrices are represented by Fibonacci and Lucas Numbers. In this study, the formulas of determinants and inverse matrices are much simpler and concise for programming and reduce the computational time.

\section{Introduction}

The good properties and structure of the circulant matrix are widely given in the area of coding theory, image processing, signal system, and cybernetics [1-4]. For instance, Carrasquinha et al. [5] propose an innovative method for image reconstruction based on circulant matrices. This method significantly reduces the computational time than the principal component analysis (PCA), the discrete Fourier transform (DFT), and the randomized singular value decomposition (RSVD). By using the circulant matrices, Nguyen and Kim [6] get a new construction method of entanglement-assisted quantum error correction code (EAQECC). The circulant matrix also plays an important role in cryptography. Yu [7] investigates the security of a cryptosystem which is based on compressed sensing. The cryptosystem encrypts a plaintext with a secret circulant matrix and transmits the ciphertext over a wireless channel. When the $\mathrm{EA}$ and $\mathrm{AE}$ robustness and $\mathrm{X}$-robustness of interval circulant matrices over max-min algebra are defined, Myšková and Plavka [8] give polynomial algorithms for checking these types of robustness and X-robustness. By comparing the coefficients in the expansion of the permanent with those in the expansion of the determinant of a three-line circulant matrix, Poi et al. [9] solve a conjecture stated in the minimality of GT systems. Using block circulant matrices, Dong [10] considers the GPU acceleration of deep neural networks.

Recently, many scholars focus on the norms, determinants, and inverses of circulant and r-circulant matrices whose entries are special integer sequences. In particular, various algorithms for the determinants and inverses of nonsingular circulant matrices and $r$-circulant matrices were gained. For example, Shen and Cen [11] get the upper and lower bounds for the spectral norms of $\mathrm{r}$-circulant matrices in the forms $A=\operatorname{Circ}_{r}\left(F_{0}, F_{1}, F_{2}, \ldots, F_{n-1}\right)$ and $B=\operatorname{Circ}_{r}\left(L_{0}, L_{1}, L_{2}, \ldots, L_{n-1}\right)$ and obtain some bounds for the spectral norms of Kronecker and Hadamard products of $A$ and $B$. Shen et al. [12] prove that circulant matrices $A=$ $\operatorname{Circ}\left(F_{0}, F_{1}, F_{2}, \ldots, F_{n-1}\right)$ is invertible for $n>2$ and $B=\operatorname{Circ}\left(L_{0}, L_{1}, L_{2}, \ldots, L_{n-1}\right)$ is invertible for any positive integer $n$. The formulas for calculating the determinants and inverses of $A$ and $B$ are derived, respectively. Afterwards, Bozkurt and Tam [13] also find the explicit determinants and inverses of circulant matrices with Jacobsthal and 
Jacobsthal-Lucas numbers by the same matrix decompositions. Then, Bozkurt and Tam [14] analyze the determinants and inverses of $r$-circulant matrices with generalized linear recursive sequence and give more accurate bounds for spectral norms of $r$-circulant matrix involving Fibonacci and Lucas numbers. Yazlik and Taskara [15] summarize the computing method for inverse of circulant matrices, in which the elements include all second-order sequences and polynomials. Zheng and Shon [16] consider the invertibility of generalized Lucas skew circulant matrix and give the determinant and inverse matrix by constructing the transformation matrices. Carmona et al. [17] propose the necessary and sufficient conditions for the invertibility of some circulant matrices that depend on three parameters and compute explicitly the inverse of a general symmetric, circulant, and tridiagonal matrix. Hong and Jing [18] investigate the invertibility of the Tribonacci r-circulant matrix and show the determinant and the inverse matrix based on constructing the transformation matrices. He et al. [19] and Türkmen and Gökbas [20] study the spectral norm of r-circulant matrices with Fibonacci and Lucas numbers and Pell and Pell-Lucas numbers, respectively. When certain circulant matrices having $k$ ones and $k+1$ zeros in the first row are nonsingular, Chen [21] proves the conditions which nonsingular matrices satisfy.

Shen et al. [12] give the techniques for calculating the determinants and inverses of circulant matrices including Fibonacci and Lucas numbers. The authors construct two matrices $P$ and $Q_{1}$, which are convenient to obtain the determinant. Then, simplify the circulant matrix $A$ to a simple form after promultiplied by $P$ and postmultiplied by $Q_{1}$. After that, they introduce an upper triangular matrix $Q_{2}$ and reduce the product of $P A Q_{1} Q_{2}$ to a direct sum of two matrices and finally obtain the inverse matrices of $A=\operatorname{Circ}\left(F_{0}, F_{1}, F_{2}, \ldots, F_{n-1}\right) \quad$ and $\quad B=\operatorname{Circ}\left(L_{0}, L_{1}\right.$, $\left.L_{2}, \ldots, L_{n-1}\right)$.

From the above papers, we find that there are no simple ways to compute the determinant and inverse of $r$-circulant matrices. The $r$-circulant matrix $A_{n}$ can be written as a polynomial about basic $r$-circulant matrix, $\pi=\operatorname{Circ}_{r}(0,1,0, \ldots, 0)$. By simplifying the subtraction of $A_{n}$ and $A_{n} \pi$, we obtain a special factorization $(E-\alpha \pi)(E-\beta \pi)$. On the properties of Fibonacci and Lucas numbers, we use this new method of matrix decomposition to express the determinants and inverses of $r$-circulant matrices where the elements are Fibonacci and Lucas numbers. The nonsingular condition of the $r$-circulant matrices is obtained. The expression of the inverse is determined by the first row of the inverse matrix. These computing formulas are simpler than the related studies. Therefore, they are more useful for practical application.

The arrangement of this paper is as follows. In Section 2, we give the preliminary knowledge and lemmas. In Section 3, the determinants and inverses of $r$-circulant matrix with Fibonacci numbers are obtained. In Section 4, the determinants and inverses of $r$-circulant matrix with Lucas numbers are obtained. Section 5 presents numerical examples.

\section{Preliminaries}

For $n \in N_{0}$, the Fibonacci and Lucas numbers are defined by $F_{n+2}=F_{n+1}+F_{n}$ and $L_{n+2}=L_{n+1}+L_{n}$ under the initial conditions $F_{0}=0, F_{1}=1$ and $L_{0}=2, L_{1}=1$, respectively. Let $\alpha=(1+\sqrt{5}) / 2$ and $\beta=(1-\sqrt{5}) / 2$ be the roots of the characteristic equation $x^{2}-x-1=0$; then, the Binet formulas of the sequences $\left\{F_{n}\right\}$ and $\left\{L_{n}\right\}$ have the form

$$
\begin{aligned}
& F_{n}=\frac{\alpha^{n}-\beta^{n}}{\alpha-\beta}, \\
& L_{n}=\alpha^{n}+\beta^{n} .
\end{aligned}
$$

Definition 1 (see [8]). For any given $c_{0}, c_{1}, \ldots, c_{n-1} \in C$, the $n \times n r$-circulant matrix $C_{n}=\operatorname{Circ}_{r}\left(c_{0}, c_{1}, \ldots, c_{n-1}\right)$ is defined by

$$
C_{n}=\left(\begin{array}{cccccc}
c_{0} & c_{1} & c_{2} & \cdots & c_{n-2} & c_{n-1} \\
r c_{n-1} & c_{0} & c_{1} & \cdots & c_{n-3} & c_{n-2} \\
\vdots & \vdots & \vdots & \ddots & \vdots & \vdots \\
r c_{2} & r c_{3} & r c_{4} & \cdots & c_{0} & c_{1} \\
r c_{1} & r c_{2} & r c_{3} & \cdots & r c_{1} & c_{0}
\end{array}\right),
$$

where $r=1$ and $C_{n}$ is a classical circulant matrix, and reduced to $C_{n}=\operatorname{Circ}\left(c_{0}, c_{1}, \ldots, c_{n-1}\right)$.

Let $\quad \pi=\operatorname{Circ}_{r}(0,1,0, \ldots, 0)$; we have $\pi^{i}=\operatorname{Circ}(0, \ldots, 0,1,0, \ldots, 0)$ and $\pi^{n}=r E$. If $\pi^{0}=E$, we obtain

$$
C_{n}=c_{0} E+c_{1} \pi+c_{2} \pi^{2}+\cdots+c_{n-1} \pi^{n-1} .
$$

Lemma 1. $\operatorname{det}(a E-b \pi)=a^{n}-r b^{n}$.

Lemma 2. Inverse matrix of the r-circulant matrix is a $r$-circulant matrix.

Lemma 3. Product of $r$-circulant matrices is a $r$-circulant matrix.

Lemma 4 (see [12]). Let $A_{n}=\operatorname{Circ}\left(F_{1}, F_{2}, \ldots, F_{n}\right)$ be a circulant matrix, and we have

$$
\operatorname{det} A_{n}=\left(1-F_{n+1}\right)^{n-1}+F_{n}^{n-2} \sum_{k=1}^{n-1} F_{k}\left(\frac{1-F_{n+1}}{F_{n}}\right)^{k-1},
$$

where $F_{n}$ is the nth Fibonacci number.

Lemma 5 (see [12]). Let $A_{n}=\operatorname{Circ}\left(F_{1}, F_{2}, \ldots, F_{n}\right)(n>2)$ be a circulant matrix; we have 


$$
\begin{aligned}
A_{n}^{-1}= & \frac{1}{f_{n}} \operatorname{Circ}\left(1+\sum_{i=1}^{n-2} \frac{F_{n-i} F_{n}^{i-1}}{\left(F_{1}-F_{n+1}\right)^{i}},-1+\sum_{i=1}^{n-2} \frac{F_{n-1-i} F_{n}^{i-1}}{\left(F_{1}-F_{n+1}\right)^{i}},-\frac{1}{F_{1}-F_{n+1}},\right. \\
& \left.-\frac{F_{n}}{\left(F_{1}-F_{n+1}\right)^{2}},-\frac{F_{n}^{2}}{\left(F_{1}-F_{n+1}\right)^{3}}, \ldots,-\frac{F_{n}^{n-3}}{\left(F_{1}-F_{n+1}\right)^{n-2}}\right)
\end{aligned}
$$

where $f_{n}=F_{1}-F_{n}+\sum_{k=1}^{n-2} F_{k}\left(F_{n} / F_{1}-F_{n+1}\right)^{n-(k+1)}$.

Lemma 6 (see [12]). Let $B_{n}=\operatorname{Circ}\left(L_{1}, L_{2}, \ldots, L_{n}\right)$ be a circulant matrix; the determinant of $B_{n}$ is

$$
\operatorname{det} B_{n}=\left(1-L_{n+1}\right)^{n-1}+\left(L_{n}-2\right)^{n-2} \sum_{k=1}^{n-1}\left(L_{k+2}-3 L_{k+1}\right)\left(\frac{1-L_{n+1}}{L_{n}-2}\right)^{k-1}
$$

where $L_{n}$ is the nth Lucas number.

Lemma 7 (see [12]). Let $B_{n}=\operatorname{Circ}\left(L_{1}, L_{2}, \ldots, L_{n}\right)$ be a circulant matrix; the determinant of $B_{n}$ is

$$
\begin{aligned}
B_{n}^{-1}= & \frac{1}{l_{n}} \operatorname{Circ}\left(1+\sum_{i=1}^{n-2} \frac{\left(L_{n+2-i}-3 L_{n+1-i}\right)\left(L_{n}-2\right)^{i-1}}{\left(L_{1}-L_{n+1}\right)^{i}},-3\right. \\
& \left.+\sum_{i=1}^{n-2} \frac{\left(L_{n+1-i}-3 L_{n-i}\right)\left(L_{n}-2\right)^{i-1}}{\left(L_{1}-L_{n+1}\right)^{i}},-\frac{5}{L_{1}-L_{n+1}},-\frac{5\left(L_{n}-2\right)}{\left(L_{1}-L_{n+1}\right)^{2}},-\frac{5\left(L_{n}-2\right)^{2}}{\left(L_{1}-L_{n+1}\right)^{3}}, \ldots,-\frac{5\left(L_{n}-2\right)^{n-3}}{\left(L_{1}-L_{n+1}\right)^{n-2}}\right),
\end{aligned}
$$

$\begin{aligned} & \text { where } \\ & +\sum_{k=1}^{n-2}\left(L_{k+2}-3 L_{k+1}\right)\left(L_{n}-2 / L_{1}-L_{n+1}\right)^{n-(k+1)}\end{aligned} l_{n}=L_{1}-3 L_{n}$

Lemma 8 (see [22]). Let $C=\operatorname{circ}\left\{{ }_{k}\left(c_{0}, c_{1}, c_{2}, \ldots, c_{n-1}\right)\right\}$ be an invertible matrix with complex entries. Then, $C^{-1}=\operatorname{circ}\left\{{ }_{k}\left(c_{0}^{\prime}, c_{1}^{\prime}, c_{2}^{\prime}, \ldots, c_{n-1}^{\prime}\right)\right\}$, where $\left(c_{0}^{\prime}, c_{1}^{\prime}, c_{2}^{\prime}, \ldots, c_{n-1}^{\prime}\right)$ is the unique solution of the following system of linear equations:

$$
C\left(\begin{array}{c}
x_{0} \\
k x_{n-1} \\
\vdots \\
k x_{1}
\end{array}\right)=\left(\begin{array}{c}
1 \\
0 \\
\vdots \\
0
\end{array}\right)
$$

Lemma 9 (see [23]). Let $C=\operatorname{circ}_{n}\left\{{ }_{k}\left(c_{0}, c_{1}, c_{2}, \ldots, c_{n-1}\right)\right\}$ be a nonsingular matrix with complex entries. Then, $C^{-1}=\operatorname{circ}_{n}\left\{{ }_{k}\left(c_{0}^{\prime}, c_{1}^{\prime}, c_{2}^{\prime}, \ldots, c_{n-1}^{\prime}\right)\right\}$, where $\left(c_{0}^{\prime}, c_{1}^{\prime}, c_{2}^{\prime}, \ldots, c_{n-1}^{\prime}\right)$ is the unique solution of the following system of linear equations:

$$
C\left(\begin{array}{c}
x_{j-1} \\
\vdots \\
x_{1} \\
x_{0} \\
k x_{n-1} \\
\vdots \\
k x_{j}
\end{array}\right)=\left(\begin{array}{c}
0 \\
\vdots \\
0 \\
1 \\
0 \\
\vdots \\
0
\end{array}\right) \leftarrow j, j=\overline{1, n} .
$$

\section{Determinant and Inverse of r-Circulant Matrix with Fibonacci Numbers}

In this section, $A_{n}=\operatorname{Circ}_{r}\left(F_{1}, F_{2}, \ldots, F_{n}\right)$ is a $r$-circulant matrix. A determinant formula for the $r$-circulant matrix $A_{n}$ is obtained. We prove that $A_{n}$ is an invertible matrix and obtain the inverse of the $r$-circulant matrix $A_{n}$.

Theorem 1. When $r \neq \alpha^{-n}$ and $r \neq \beta^{-n}$, the determinant of $A_{n}$ is 


$$
\operatorname{det} A_{n}=\frac{\left(1-r F_{n+1}\right)^{n}-r^{n+1} F_{n}^{n}}{1+(-1)^{n} r^{2}-r L_{n}},
$$

where $F_{n}$ and $L_{n}$ are the nth Fibonacci number and Lucas number, respectively.

Proof. From equation (3), $A_{n}$ is represented by

$$
A_{n}=F_{1} E+F_{2} \pi+F_{3} \pi^{2}+\cdots+F_{n} \pi^{n-1} .
$$
(7) is

Right multiplication matrix $\pi$ on both sides of equation

$$
A_{n} \pi=F_{1} \pi+F_{2} \pi^{2}+F_{3} \pi^{3}+\cdots+F_{n} \pi^{n} .
$$

From (11) and (12), we obtain

$$
\begin{aligned}
A_{n}-A_{n} \pi= & \left(F_{1}-r F_{n}\right) E+\left(F_{2}-F_{1}\right) \pi \\
& +\left(F_{3}-F_{2}\right) \pi^{2}+\cdots+\left(F_{n}-F_{n-1}\right) \pi^{n-1} .
\end{aligned}
$$

Because $F_{0}=0, F_{1}=1$, and $F_{n+2}=F_{n+1}+F_{n}$, equation (11) and equation (13) become

$$
\begin{aligned}
A_{n}-A_{n} \pi= & \left(F_{1}-r F_{n}\right) E+F_{0} \pi+F_{1} \pi^{2}+\cdots+F_{n-2} \pi^{n-1} \\
= & \left(1-r F_{n}-r F_{n-1}\right) E-r F_{n} \pi \\
& +\left(F_{1} E+F_{2} \pi+F_{3} \pi^{2}+\cdots+F_{n} \pi^{n-1}\right) \pi^{2} \\
= & \left(1-r F_{n+1}\right) E-r F_{n} \pi+A_{n} \pi^{2} .
\end{aligned}
$$

Thus,

$$
A_{n}\left(E-\pi-\pi^{2}\right)=\left(1-r F_{n+1}\right) E-r F_{n} \pi .
$$

When $r \neq \alpha^{-n}, r \neq \beta^{-n}$, and $L_{n}^{n}=\alpha^{n}+\beta^{n}$, we obtain

$$
\begin{aligned}
\operatorname{det}\left(E-\pi-\pi^{2}\right) & =\operatorname{det}(E-\alpha \pi)(E-\beta \pi) \\
& =\left(1-r \alpha^{n}\right)\left(1-r \beta^{n}\right) \\
& =1+(-1)^{n} r^{2}-r L_{n} \neq 0 .
\end{aligned}
$$

From Lemma 1, the determinant of equation (15) is

$$
\begin{aligned}
\operatorname{det} A_{n} \operatorname{det}\left(E-\pi-\pi^{2}\right) & =\operatorname{det} A_{n}\left(E-\pi-\pi^{2}\right) \\
& =\operatorname{det}\left(\left(1-r F_{n+1}\right) E-r F_{n} \pi\right) \\
& =\left(1-r F_{n+1}\right)^{n}-r^{n+1} F_{n}^{n} .
\end{aligned}
$$

Hence, the determinant of $A_{n}$ is

$$
\operatorname{det} A_{n}=\frac{\left(1-r F_{n+1}\right)^{n}-r^{n+1} F_{n}^{n}}{1+(-1)^{n} r^{2}-r L_{n}} .
$$

Corollary 1. Under the assumption of Theorem 1, when $r=1$, the determinant of $A_{n}$ is

$$
\operatorname{det} A_{n}=\frac{\left(1-F_{n+1}\right)^{n}-F_{n}^{n}}{1+(-1)^{n}-L_{n}}
$$

where $F_{n}$ and $L_{n}$ are the nth Fibonacci number and Lucas number, respectively.

Proof. From the proof of Theorem 1, we have

$$
A_{n}\left(E-\pi-\pi^{2}\right)=\left(1-F_{n+1}\right) E-F_{n} \pi .
$$

When $r=1$, we get $1 \neq \alpha^{-n}$ and $1 \neq \beta^{-n}$; thus,

$$
\begin{aligned}
\operatorname{det}\left(E-\pi-\pi^{2}\right) & =\operatorname{det}(E-\alpha \pi)(E-\beta \pi) \\
& =\left(1-\alpha^{n}\right)\left(1-\beta^{n}\right) \\
& =1+(-1)^{n}-L_{n} \neq 0 \\
\operatorname{det}\left(\left(1-F_{n+1}\right) E-F_{n} \pi\right) & =\left(1-F_{n+1}\right)^{n}-F_{n}^{n} .
\end{aligned}
$$

Hence, we obtain

$$
\operatorname{det} A_{n}=\frac{\left(1-F_{n+1}\right)^{n}-F_{n}^{n}}{1+(-1)^{n}-L_{n}} .
$$

Obviously, the computation formula of Corollary 1 is simpler than Lemma 4.

Corollary 2. Under the assumption of Theorem 1, when $\left(1-r F_{n+1} / r F_{n}\right)^{n} \neq r, A_{n}$ is an invertible matrix.

Proof. Since $\left(1-r F_{n+1} / r F_{n}\right)^{n} \neq r$, we have

$$
\operatorname{det}\left(\left(1-r F_{n+1}\right) E-r F_{n} \pi\right)=\left(1-r F_{n+1}\right)^{n}-r^{n+1} F_{n}^{n} \neq 0 .
$$

Then,

$$
\operatorname{det} A_{n}=\frac{\left(1-r F_{n+1}\right)^{n}-r^{n+1} F_{n}^{n}}{1+(-1)^{n} r^{2}-r L_{n}} \neq 0 .
$$

Therefore, $A_{n}$ is an invertible matrix.

Theorem 2. Under the assumption of Theorem 1, if $\left(1-r F_{n+1} / r F_{n}\right)^{n} \neq r$, the inverse of $A_{n}$ is

$$
A_{n}^{-1}=\frac{x^{n-1}}{x^{n}+(-1)^{n-1} r y^{n}} \operatorname{Circ}_{r}\left(y_{1}, y_{2}, y_{3}, \ldots, y_{n}\right) \text {, }
$$

where $\quad x=1-r F_{n+1}, \quad y=-r F_{n}, \quad x y \neq 0$, $y_{1}=1-r(-y / x)^{n-2}-r(-y / x)^{n-1}$,

$y_{2}=-1-(-y / x)-r(-y / x)^{n-1}$, and $y_{i}=(-y / x)^{i-1}-(-y / x)^{i-2}-(-y / x)^{i-3}(i=3,4, \ldots, n)$.

Proof. From the proof of Theorem 1, we have

$$
A_{n}\left(E-\pi-\pi^{2}\right)=\left(1-r F_{n+1}\right) E-r F_{n} \pi .
$$

When $\left(1-r F_{n+1} / r F_{n}\right)^{n} \neq r$, let $\quad x=1-r F_{n+1} \quad$ and $y=-r F_{n}, x y \neq 0$. From Lemma 2, the $r$-circulant matrix $x E+y \pi$ is an invertible $r$-circulant matrix. From Lemmas 8 and $9,(x E+y \pi)^{-1}=\operatorname{Circ}_{r}\left(x_{1}, x_{2}, x_{3}, \ldots, x_{n}\right)$, where $x_{1}, x_{2}$, $x_{3}, \cdots, x_{n}$ are the unique solutions of the following linear system: 


$$
\left(\begin{array}{ccccc}
x & y & 0 & \cdots & 0 \\
0 & x & y & \cdots & 0 \\
\vdots & \vdots & \vdots & \ddots & \vdots \\
0 & 0 & 0 & \cdots & y \\
r y & 0 & 0 & \cdots & x
\end{array}\right)\left(\begin{array}{c}
x_{1} \\
r x_{n} \\
\vdots \\
r x_{3} \\
r x_{2}
\end{array}\right)=\left(\begin{array}{c}
1 \\
0 \\
\vdots \\
0 \\
0
\end{array}\right) .
$$

Then, the solution of equation (27) is

$$
\begin{aligned}
& x_{1}=\frac{x^{n-1}}{x^{n}+(-1)^{n-1} r y^{n}}, \\
& x_{2}=\frac{x^{n-1}}{x^{n}+(-1)^{n-1} r y^{n}}\left(-\frac{y}{x}\right), \\
& x_{3}=\frac{x^{n-1}}{x^{n}+(-1)^{n-1} r y^{n}}\left(-\frac{y}{x}\right)^{2}, \\
& \vdots \\
& x_{n}=\frac{x^{n-1}}{x^{n}+(-1)^{n-1} r y^{n}}\left(-\frac{y}{x}\right)^{n-1} .
\end{aligned}
$$

Let

$$
\begin{aligned}
& \left(E-\pi-\pi^{2}\right) \operatorname{Circ}_{r}\left(1,-\frac{y}{x},\left(-\frac{y}{x}\right)^{2}, \ldots,\left(-\frac{y}{x}\right)^{n-1}\right) \\
& =\operatorname{Circ}_{r}\left(y_{1}, y_{2}, \ldots, y_{n}\right) .
\end{aligned}
$$

We obtain

$$
\begin{aligned}
& y_{1}=1-r\left(-\frac{y}{x}\right)^{n-2}-r\left(-\frac{y}{x}\right)^{n-1}, \\
& y_{2}=-1-\frac{y}{x}-r\left(-\frac{y}{x}\right)^{n-1}, \\
& y_{i}=\left(-\frac{y}{x}\right)^{i-1}-\left(-\frac{y}{x}\right)^{i-2}-\left(-\frac{y}{x}\right)^{i-3}, \quad(i=3,4, \ldots, n) .
\end{aligned}
$$

Hence, the inverse of $A_{n}$ is

$$
A_{n}^{-1}=\frac{x^{n-1}}{x^{n}+(-1)^{n-1} r y^{n}} \operatorname{Circ}_{r}\left(y_{1}, y_{2}, y_{3}, \ldots, y_{n}\right) \text {. }
$$

Corollary 3. Under the assumption of Theorem 2, when $\left(1-F_{n+1} / F_{n}\right)^{n} \neq 1$ and $r=1$, the inverse of $A_{n}$ is

$$
\begin{aligned}
A_{n}^{-1}= & \frac{x^{n-1}}{x^{n}+(-1)^{n-1} y^{n}} \operatorname{Circ}_{r}\left(1-r\left(-\frac{y}{x}\right)^{n-2}-r\left(-\frac{y}{x}\right)^{n-1},-1\right. \\
& -\frac{y}{x}-r\left(-\frac{y}{x}\right)^{n-1},\left(-\frac{y}{x}\right)^{2}-\left(-\frac{y}{x}\right)-1, \ldots,\left(-\frac{y}{x}\right)^{i-1} \\
& \left.-\left(-\frac{y}{x}\right)^{i-2}-\left(-\frac{y}{x}\right)^{i-3}\right),
\end{aligned}
$$

where $x=1-F_{n+1}$ and $y=-F_{n}, x y \neq 0$.

Proof. Under the condition of Corollary 2, when $\left(1-F_{n+1} / F_{n}\right)^{n} \neq 1$ and $r=1$, then $A_{n}$ is an invertible circulant matrix. From Theorem 2, we obtain

$$
\begin{aligned}
A_{n}^{-1}= & \frac{x^{n-1}}{x^{n}+(-1)^{n-1} y^{n}} \operatorname{Circ}_{r}\left(1-r\left(-\frac{y}{x}\right)^{n-2}-r\left(-\frac{y}{x}\right)^{n-1},\right. \\
& -1-\frac{y}{x}-r\left(-\frac{y}{x}\right)^{n-1},\left(-\frac{y}{x}\right)^{2}-\left(-\frac{y}{x}\right) \\
& \left.-1, \ldots,\left(-\frac{y}{x}\right)^{i-1}-\left(-\frac{y}{x}\right)^{i-2}-\left(-\frac{y}{x}\right)^{i-3}\right),
\end{aligned}
$$

where $x=1-F_{n+1}$ and $y=-F_{n}, x y \neq 0$.

Obviously, the formula of Corollary 3 is simpler than Lemma 5.

\section{Determinant and Inverse of $\boldsymbol{r}$-Circulant Matrix with Lucas Numbers}

Let $B_{n}=\operatorname{Circ}_{r}\left(L_{1}, L_{2}, \ldots, L_{n}\right)$ be a $r$-circulant matrix, where $L_{n}$ is the Lucas numbers. We discuss the nonsingular conditions for $B_{n}$ and obtain the determinant formula and inverse of the $r$-circulant matrix $B_{n}$.

Theorem 3. When $r \neq \alpha^{-n}$ and $r \neq \beta^{-n}$, the determinant of $B_{n}$ is

$$
\operatorname{det} B_{n}=\frac{\left(1-r L_{n+1}\right)^{n}-r\left(r L_{n}-2\right)^{n}}{1+(-1)^{n} r^{2}-r L_{n}},
$$

where $L_{n}$ is the Lucas numbers.

Proof. By the proof of Theorem 1, we have

$$
B_{n}\left(E-\pi-\pi^{2}\right)=\left(1-r L_{n+1}\right) E+\left(2-r L_{n}\right) \pi .
$$

When $r \neq \alpha^{-n}, r \neq \beta^{-n}$, and $L_{n}=\alpha^{n}+\beta^{n}$, we obtain

$$
\operatorname{det}\left(E-\pi-\pi^{2}\right)=1+(-1)^{n} r^{2}-r L_{n} \neq 0 .
$$

From Lemma 1, we obtain

$$
\operatorname{det}\left(\left(1-r L_{n+1}\right) E+\left(2-r L_{n}\right) \pi\right)=\left(1-r L_{n+1}\right)^{n}-r\left(r L_{n}-2\right)^{n} \text {. }
$$

Hence, the determinant of $B_{n}$ is

$$
\operatorname{det} B_{n}=\frac{\left(1-r L_{n+1}\right)^{n}-r\left(r L_{n}-2\right)^{n}}{1+(-1)^{n} r^{2}-r L_{n}} .
$$

Corollary 4. Under the assumption of Theorem 3, when $r=1$, the determinant of $B_{n}$ is

$$
\operatorname{det} B_{n}=\frac{\left(1-L_{n+1}\right)^{n}-\left(L_{n}-2\right)^{n}}{1+(-1)^{n}-L_{n}},
$$


where $L_{n}$ is the nth Lucas number.

Obviously, the computation formula of Corollary 4 is simpler than Lemma 6.
Corollary 5. Under the assumption of Theorem 3, when $\left(1-r L_{n+1} / r L_{n}-2\right)^{n} \neq r, B_{n}$ is an invertible matrix.

Proof. Since $\left(1-r L_{n+1} / r L_{n}-2\right)^{n} \neq r$, we obtain

$$
\operatorname{det}\left(\left(1-r L_{n+1}\right) E+\left(2-r L_{n}\right) \pi\right)=\left(1-r L_{n+1}\right)^{n}-r\left(r L_{n}-2\right)^{n} \neq 0 .
$$

Because

$$
\operatorname{det} B_{n} \neq 0
$$

$B_{n}$ is an invertible matrix.

Theorem 4. Under the assumption of Theorem 3, if $\left(1-r L_{n+1} / r L_{n}-2\right)^{n} \neq r$, the inverse of $B_{n}$ is

$$
B_{n}^{-1}=\frac{x^{n-1}}{x^{n}+(-1)^{n-1} r y^{n}} \operatorname{Circ}_{r}\left(y_{1}, y_{2}, y_{3}, \ldots, y_{n}\right) \text {, }
$$

where $x=1-r L_{n+1}, \quad y=2-r L_{n}, \quad x y \neq 0, \quad y_{1}=1-r(-y$ $(x)^{n-2}-r(-y / x)^{n-1}, y_{2}=-1(-y / x)-r(-y / x)^{n-1}$, and $y_{i}=$ $(-y / x)^{i-1}-(-y / x)^{i-2}-(-y / x)^{i-3}(i=3,4, \ldots, n)$.

$$
\left(\begin{array}{ccccc}
x & y & 0 & \cdots & 0 \\
0 & x & y & \cdots & 0 \\
\vdots & \vdots & \vdots & \ddots & \vdots \\
0 & 0 & 0 & \cdots & y \\
r y & 0 & 0 & \cdots & x
\end{array}\right)\left(\begin{array}{c}
x_{1} \\
r x_{n} \\
\vdots \\
r x_{3} \\
r x_{2}
\end{array}\right)=\left(\begin{array}{c}
1 \\
0 \\
\vdots \\
0 \\
0
\end{array}\right) .
$$

Proof. From the proof of Theorem 3, we have

$$
B_{n}\left(E-\pi-\pi^{2}\right)=\left(1-r L_{n+1}\right) E+\left(2-r L_{n}\right) \pi .
$$

When $\left(1-r L_{n+1} / r L_{n}-2\right)^{n} \neq r$, let $x=1-r L_{n+1}$ and $y=2-r L_{n}, x y \neq 0$; from Lemma 2, the $r$-circulant matrix $x E+y \pi$ is an invertible $r$-circulant matrix. From Lemmas 8 and $9,(x E+y \pi)^{-1}=\operatorname{Circ}_{r}\left(x_{1}, x_{2}, x_{3}, \ldots, x_{n}\right)$, where $x_{1}, x_{2}$, $x_{3}, \cdots, x_{n}$ are the unique solution of the following linear system:

Then, the solution of equation (44) is

$$
\begin{aligned}
& x_{1}=\frac{x^{n-1}}{x^{n}+(-1)^{n-1} r y^{n}}, \\
& x_{2}=\frac{x^{n-1}}{x^{n}+(-1)^{n-1} r y^{n}}\left(-\frac{y}{x}\right), \\
& x_{3}=\frac{x^{n-1}}{x^{n}+(-1)^{n-1} r y^{n}}\left(-\frac{y}{x}\right)^{2}, \\
& x_{n}=\frac{x^{n-1}}{x^{n}+(-1)^{n-1} r y^{n}}\left(-\frac{y}{x}\right)^{n-1} .
\end{aligned}
$$

Since

$$
\left(E-\pi-\pi^{2}\right) \operatorname{Circ}_{r}\left(1,-\frac{y}{x},\left(-\frac{y}{x}\right)^{2}, \ldots,\left(-\frac{y}{x}\right)^{n-1}\right)=\operatorname{Circ}_{r}\left(y_{1}, y_{2}, \ldots, y_{n}\right) .
$$

We obtain

$$
\begin{aligned}
& y_{1}=1-r\left(-\frac{y}{x}\right)^{n-2}-r\left(-\frac{y}{x}\right)^{n-1}, \\
& y_{2}=-1-\frac{y}{x}-r\left(-\frac{y}{x}\right)^{n-1}, \\
& y_{i}=\left(-\frac{y}{x}\right)^{i-1}-\left(-\frac{y}{x}\right)^{i-2}-\left(-\frac{y}{x}\right)^{i-3} \quad(i=3,4, \ldots, n) .
\end{aligned}
$$

Hence, the inverse of $B_{n}$ is

$$
A^{-1}=\frac{x^{n-1}}{x^{n}+(-1)^{n-1} r y^{n}} \operatorname{Circ}_{r}\left(y_{1}, y_{2}, y_{3}, \ldots, y_{n}\right) \text {. }
$$

Corollary 6. Under the assumption of Theorem 4, when $\left(1-L_{n+1} / L_{n}-2\right)^{n} \neq 1$ and $r=1$, the inverse of $B_{n}$ is

$$
\begin{aligned}
B_{n}^{-1}= & \frac{x^{n-1}}{x^{n}+(-1)^{n-1} y^{n}} \operatorname{Circ}_{r}\left(1-r\left(-\frac{y}{x}\right)^{n-2}-r\left(-\frac{y}{x}\right)^{n-1},-1-\frac{y}{x}\right. \\
& \left.-r\left(-\frac{y}{x}\right)^{n-1},\left(-\frac{y}{x}\right)^{2}-\left(-\frac{y}{x}\right)-1, \ldots,\left(-\frac{y}{x}\right)^{i-1}-\left(-\frac{y}{x}\right)^{i-2}-\left(-\frac{y}{x}\right)^{i-3}\right),
\end{aligned}
$$




\begin{tabular}{|c|c|c|c|c|c|c|c|c|c|c|c|c|}
\hline & 1 & 2 & 3 & 4 & 5 & 6 & 7 & 8 & 9 & 10 & 11 & 12 \\
\hline 1 & $-1.4219 \mathrm{e}-2 \ldots$ & $2.3006 \mathrm{e}-209$ & 9 & 0 & 0 & 0 & 0 & 0 & 0 & 0 & 0 & 0 \\
\hline 2 & & $0-1.4219 \mathrm{e}-2 \ldots$ & $2.3006 \mathrm{e}-209$ & & 0 & 0 & 0 & 0 & 0 & 0 & 0 & 0 \\
\hline 3 & 0 & 0 & $0-1.4219 e-2 \ldots$ & $2.3006 \mathrm{e}-209$ & & 0 & 0 & 0 & 0 & 0 & 0 & 0 \\
\hline 4 & 0 & 0 & 0 & D-1.4219e-2... & 2.3006e-209 & 9 & 0 & 0 & 0 & 0 & 0 & 0 \\
\hline 5 & 0 & 0 & 0 & 0 & $0-1.4219 \mathrm{e}-2 \ldots$ & 2.3006e-209 & & 0 & 0 & 0 & 0 & 0 \\
\hline 6 & 0 & 0 & 0 & D & 0 & $0-1.4219 e-2 \ldots$ & $2.3006 \mathrm{e}-209$ & & 0 & 0 & 0 & 0 \\
\hline 7 & 0 & 0 & 0 & D & 0 & 0 & D - $1.4219 e-2 \ldots$ & 2.3006e-209 & & 0 & 0 & 0 \\
\hline 8 & 0 & 0 & 0 & 0 & 0 & 0 & 0 & $0-1.4219 e-2 \ldots$ & .. 2.3006e-20s & 0 & 0 & 0 \\
\hline 9 & 0 & 0 & 0 & & 0 & 0 & 0 & 0 & $0-1.4219 \mathrm{e}-2$. & $2.3006 \mathrm{e}-209$ & & 0 \\
\hline 10 & 0 & & 0 & 0 & 0 & 0 & 0 & 0 & 0 & $0-1.4219 \mathrm{e}-2 \ldots$ & $2.3006 \mathrm{e}-209$ & 9 \\
\hline 11 & 0 & & 0 & 0 & 0 & 0 & 0 & 0 & 0 & 0 & $-1.4219 \mathrm{e}-2 \ldots$ & ... 2.3006e-209 \\
\hline 12 & 0 & & 0 & D & 0 & 0 & 0 & 0 & 0 & 0 & 0 & $0-1.4219 e-2 \ldots$ \\
\hline
\end{tabular}

Figure 1: The result of Lemma 5.

\begin{tabular}{|c|c|c|c|c|c|c|c|c|c|c|c|c|}
\hline & 1 & 2 & 3 & 4 & 5 & 6 & 7 & 8 & 9 & 10 & 11 & 12 \\
\hline 1 & $-1.4219 \mathrm{e}-2 .$. & $2.3006 \mathrm{e}-209$ & 0 & 0 & $0-7.8929 \mathrm{e}-2 \ldots$ & .. $7.8929 \mathrm{e}-226$ & & $01.9732 e-226$ & $6-9.8661 e-2 \ldots$ & .. 9.8661e-227 & $-4.9331 e-2 \ldots$ & $2.4665 \mathrm{e}-227$ \\
\hline 2 & & $0-1.4219 \mathrm{e}-2 \ldots$ & $2.3006 \mathrm{e}-209$ & & 0 & $0-7.8929 \mathrm{e}-2 \ldots$ & ... $7.8929 \mathrm{e}-226$ & & $01.9732 \mathrm{e}-226$ & $6-9.8661 \mathrm{e}-2 \ldots$ & $9.8661 \mathrm{e}-227$ & $7-4.9331 e-2 \ldots$ \\
\hline 3 & & 0 & $0-1.4219 e-2 \ldots$ & $2.3006 \mathrm{e}-209$ & 9 & & $0-7.8929 \mathrm{e}-2 \ldots$ & ... $7.8929 \mathrm{e}-226$ & & 0 1.9732e-226 & $-9.8661 e-2 \ldots$ & .. 9.8661e-227 \\
\hline 4 & & 0 & $0 \quad 0$ & $0-1.4219 e-2 \ldots$ & $2.3006 \mathrm{e}-209$ & & $0 \quad 0$ & $0-7.8929 \mathrm{e}-2 \ldots$ & $7.8929 \mathrm{e}-226$ & & $1.9732 \mathrm{e}-226$ & $6-9.8661 e-2 \ldots$ \\
\hline 5 & & 0 & 0 & 0 & $0-1.4219 e-2 \ldots$ & 2.3006e-209 & & 0 & $0-7.8929 e-2 \ldots$ & 7.8929e-226 & & $01.9732 \mathrm{e}-226$ \\
\hline 6 & & 0 & 0 & 0 & 0 & $0-1.4219 e-2 \ldots$ & ... $2.3006 \mathrm{e}-209$ & 9 & 0 & $0-7.8929 \mathrm{e}-2 . .$. & . $7.8929 \mathrm{e}-226$ & 6 \\
\hline 7 & 0 & 0 & 0 & 0 & 0 & & $0-1.4219 e-2 \ldots$ & ... 2.3006e-209 & & 0 & $-7.8929 e-2 \ldots$ & .. $7.8929 \mathrm{e}-226$ \\
\hline 8 & 0 & 0 & 0 & 0 & 0 & & 0 & $0-1.4219 \mathrm{e}-2 .$. & $2.3006 \mathrm{e}-209$ & 0 & & $0-7.8929 \mathrm{e}-2 \ldots$ \\
\hline 9 & 0 & 0 & 0 & 0 & 0 & & 0 & 0 & $0-1.4219 e-2 \ldots$ & 2.3006e-209 & 0 & 0 \\
\hline 10 & 0 & 0 & 0 & 0 & 0 & & 0 & 0 & 0 & $0-1.4219 e-2 \ldots$ & $2.3006 \mathrm{e}-209$ & 9 \\
\hline 11 & 0 & 0 & 0 & 0 & 0 & & 0 & 0 & 0 & 0 & $-1.4219 e-2 \ldots$ & ... 2.3006e-209 \\
\hline 12 & 0 & 0 & 0 & 0 & 0 & & 0 & 0 & 0 & 0 & 0 & $0-1.4219 e-2 .$. \\
\hline
\end{tabular}

Figure 2: The result of Corollary 3.

where $x=1-L_{n+1}$ and $y=2-L_{n}, x y \neq 0$.

Proof. Under the condition of Corollary 5, when $\left(1-L_{n+1} / L_{n}-2\right)^{n} \neq 1$ and $r=1$, then $B_{n}$ is an invertible circulant matrix. From proof of Theorem 4, we obtain

$$
\begin{aligned}
B_{n}^{-1}= & \frac{x^{n-1}}{x^{n}+(-1)^{n-1} y^{n}} \operatorname{Circ}_{r}\left(1-r\left(-\frac{y}{x}\right)^{n-2}-r\left(-\frac{y}{x}\right)^{n-1},\right. \\
& \left.-1-\frac{y}{x}-r\left(-\frac{y}{x}\right)^{n-1},\left(-\frac{y}{x}\right)^{2}-\left(-\frac{y}{x}\right)-1, \ldots,\left(-\frac{y}{x}\right)^{i-1}-\left(-\frac{y}{x}\right)^{i-2}-\left(-\frac{y}{x}\right)^{i-3}\right),
\end{aligned}
$$

where $x=1-L_{n+1}$ and $y=2-L_{n}, x y \neq 0$. Obviously, the formula of Corollary 6 is simpler than Lemma 7.

\section{Numerical Examples}

Next, we further discuss our conclusion through numerical simulation.

Example 1. When $r=1$ and $n=1000$, we obtain the inverse matrix of $A_{n}$ by Lemma 5 and Corollary 3.

Example 2. When $r=1$ and $n=1000$, we obtain the inverse matrix of $B_{n}$ by Lemma 7 and Corollary 6 .

Examples 1 and 2 calculate the inverse of a matrix for 1000 orders. Due to the limited space, we only cut 12 orders. Through Figures 1-4, we can find that our conclusion is more accurate than Lemmas 5 and 7 in the process of numerical simulation. At the same time, in the process of practical application, our conclusion will further reduce the error.

Example 3. When $r=1$, we compare the running time of Lemma 4 and Corollary 1 in milliseconds (ms).

Example 4. When $r=1$, we compare the running time of Lemma 6 and Corollary 4 in milliseconds (ms).

Through calculating, it can be concluded that the time complexity of these formulas in Lemmas 4-7 are $O\left(n^{2}\right)$. And the time complexity of formulas in Corollaries $1-6$ are $O(n)$. This new method of matrix decomposition reduces computation by an order of magnitude. We also find that the computation time is greatly reduced through programming with $C$ language. From Tables 1 and 2, the same conclusions are easily found. We calculate the inverse matrices by MATLAB; when $n \geqslant 1000$, the expression for the inverse matrices are more accurate than Lemmas 5 and 7. 


\begin{tabular}{|c|c|c|c|c|c|c|c|c|c|c|c|c|}
\hline & 1 & 2 & 3 & 4 & 5 & 6 & 7 & 8 & 9 & 10 & 11 & 12 \\
\hline 1 & $-6.3588 \mathrm{e}-2 .$. & $1.0289 \mathrm{e}-209$ & 0 & 0 & 0 & 0 & 0 & 0 & 0 & 0 & 0 & 0 \\
\hline 2 & 0 & $-6.3588 e-2$ & . 1.0289e-209 & 0 & 0 & 0 & 0 & 0 & 0 & 0 & 0 & 0 \\
\hline 3 & 0 & & $0-6.3588 \mathrm{e}-2 \ldots$ & $1.0289 \mathrm{e}-209$ & & 0 & 0 & 0 & 0 & 0 & 0 & 0 \\
\hline 4 & 0 & 0 & & $0-6.3588 e-2 \ldots$ & ... 1.0289e-209 & 0 & 0 & 0 & 0 & 0 & 0 & 0 \\
\hline 5 & 0 & 0 & 0 & & $0-6.3588 e-2 \ldots$ & ... 1.0289e-209 & 0 & 0 & 0 & 0 & 0 & 0 \\
\hline 6 & 0 & 0 & 0 & 0 & 0 & $0-6.3588 e-2 \ldots$ & . $1.0289 \mathrm{e}-209$ & & 0 & 0 & 0 & 0 \\
\hline 7 & 0 & 0 & 0 & 0 & 0 & 0 & $0-6.3588 e-2 \ldots$ & .. $1.0289 \mathrm{e}-209$ & 0 & 0 & 0 & 0 \\
\hline 8 & 0 & 0 & 0 & 0 & 0 & 0 & 0 & $0-6.3588 e-2 \ldots$ & ... $1.0289 \mathrm{e}-209$ & 0 & 0 & 0 \\
\hline 9 & 0 & 0 & 0 & 0 & 0 & 0 & 0 & 0 & $0-6.3588 e-2 \ldots$ & $1.0289 \mathrm{e}-209$ & 0 & 0 \\
\hline 10 & 0 & 0 & 0 & 0 & 0 & 0 & 0 & 0 & 0 & $-6.3588 \mathrm{e}-2$ & $1.0289 \mathrm{e}-209$ & 0 \\
\hline 11 & 0 & 0 & 0 & 0 & 0 & 0 & 0 & 0 & 0 & & $0-6.3588 \mathrm{e}-2 \ldots$ & $1.0289 \mathrm{e}-209$ \\
\hline 12 & 0 & 0 & 0 & 0 & 0 & 0 & 0 & 0 & 0 & 0 & & $-6.3588 \mathrm{e}-2$. \\
\hline
\end{tabular}

Figure 3: The result of Lemma 7.

\begin{tabular}{|c|c|c|c|c|c|c|c|c|c|c|c|c|}
\hline & 1 & 2 & 3 & 4 & 5 & 6 & 7 & 8 & 9 & 10 & 11 & 12 \\
\hline 1 & $-6.3588 \mathrm{e}-2$. & $1.0289 \mathrm{e}-209$ & $97.0596 \mathrm{e}-226$ & $6-7.0596 e-2 \ldots$ & .. $3.5298 \mathrm{e}-226$ & $5-1.7649 e-2 \ldots$ & & $0-1.7649 \mathrm{e}-2 \ldots$ & . $4.4123 \mathrm{e}-227$ & $7-4.4123 e-2 \ldots$ & & $0-1.1031 e-2 \ldots$ \\
\hline 2 & & $-6.3588 \mathrm{e}-2$ & ... $1.0289 \mathrm{e}-209$ & $97.0596 \mathrm{e}-226$ & $6-7.0596 e-2 \ldots$ & $3.5298 \mathrm{e}-226$ & $5-1.7649 \mathrm{e}-2 \ldots$ & & $-1.7649 e-2 \ldots$ & $4.4123 \mathrm{e}-227$ & $-4.4123 e-2 \ldots$ & 0 \\
\hline 3 & 0 & & $0-6.3588 \mathrm{e}-2 \ldots$ & $1.0289 \mathrm{e}-209$ & $97.0596 \mathrm{e}-226$ & $5-7.0596 e-2 \ldots$ & $3.5298 \mathrm{e}-226$ & $6-1.7649 \mathrm{e}-2 \ldots$ & & $0-1.7649 \mathrm{e}-2 \ldots$ & $4.4123 \mathrm{e}-227$ & $7-4.4123 e-2 \ldots$ \\
\hline 4 & 0 & & $0 \quad 0$ & $0-6.3588 e-2 \ldots$ & $1.0289 \mathrm{e}-209$ & $7.0596 \mathrm{e}-226$ & $5-7.0596 e-2 \ldots$ & ... $3.5298 \mathrm{e}-226$ & $5-1.7649 e-2 \ldots$ & & $-1.7649 \mathrm{e}-2 \ldots$ & $4.4123 e-227$ \\
\hline 5 & 0 & & 0 & $0 \quad 0$ & $0-6.3588 \mathrm{e}-2 \ldots$ & $1.0289 \mathrm{e}-209$ & $7.0596 \mathrm{e}-226$ & $6-7.0596 \mathrm{e}-2 . .$. & $3.5298 \mathrm{e}-226$ & $5-1.7649 \mathrm{e}-2 .$. & & $0-1.7649 e-2 \ldots$ \\
\hline 6 & 0 & & 0 & 0 & $0 \quad 0$ & $-6.3588 e-2 \ldots$ & $1.0289 \mathrm{e}-209$ & $97.0596 \mathrm{e}-226$ & $-7.0596 e-2 \ldots$ & $3.5298 \mathrm{e}-226$ & $-1.7649 \mathrm{e}-2 \ldots$ & 0 \\
\hline 7 & 0 & & 0 & 0 & 0 & 0 & $-6.3588 \mathrm{e}-2 \ldots$ & $1.0289 \mathrm{e}-209$ & $7.0596 e-226$ & $5-7.0596 e-2 \ldots .3$ & $3.5298 \mathrm{e}-226$ & $6-1.7649 e-2 \ldots$ \\
\hline 8 & 0 & & 0 & 0 & 0 & 0 & & $0-6.3588 \mathrm{e}-2 \ldots$ & . $1.0289 \mathrm{e}-209$ & $97.0596 \mathrm{e}-226$ & $-7.0596 e-2 \ldots$ & $3.5298 \mathrm{e}-226$ \\
\hline 9 & 0 & 0 & 0 & 0 & 0 & 0 & 0 & $0 \quad 0$ & $-6.3588 e-2 \ldots$ & $1.0289 \mathrm{e}-209$ & $7.0596 \mathrm{e}-226$ & $6-7.0596 e-2 \ldots$ \\
\hline
\end{tabular}

FIgURE 4: The result of Corollary 6.

Table 1: Running time comparison of Lemma 4 and Corollary 1.

\begin{tabular}{lcc}
\hline$n$ & Lemma 4 & Corollary 1 \\
\hline 20 & 0 & 0 \\
25 & 31 & 16 \\
30 & 172 & 109 \\
35 & 2278 & 1201 \\
36 & 2855 & 1996 \\
37 & 4758 & 3276 \\
38 & 7550 & 5164 \\
\hline
\end{tabular}

TABle 2: Running time comparison of Lemma 6 and Corollary 4.

\begin{tabular}{lcc}
\hline$n$ & Lemma 6 & Corollary 4 \\
\hline 20 & 0 & 0 \\
25 & 47 & 15 \\
30 & 281 & 109 \\
35 & 3073 & 1186 \\
36 & 4976 & 1903 \\
37 & 8112 & 3026 \\
38 & 12979 & 4992 \\
\hline
\end{tabular}

\section{Conclusion}

In this paper, we use a new matrix decomposition method to solve the determinant and inverse $m$ of the $r$-circulant matrix with Fibonacci sequence and Lucas sequence. Some simpler forms are obtained and the corresponding proofs are given. Through numerical examples, we find that the new formulas not only reduce the computation time of determinant but also increase the accuracy of solving inverse matrix and are more conducive to the application of real life.

\section{Data Availability}

The data used to support the findings of this study are included within the article. 


\section{Conflicts of Interest}

The authors declare that there are no conflicts of interest regarding the publication of this paper.

\section{Acknowledgments}

This work was supported by the Modernization of Urban and Rural Governance Research Center of Chengdu Key Research Base of Philosophy and Social Sciences (CXZL202104), Key Research Base of Philosophy and Social Sciences for Colleges and Universities in Sichuan Province (KJJR2019-004), Talent Introduction Project of Xihua University (w202247), and Applied Fundamental Research Program of Sichuan Province (no. 2018JY0503).

\section{References}

[1] J. F. Coeurjolly and E. Porcu, "Fast and exact simulation of complex-valued stationary Gaussian processes through embedding circulant matrix," Journal of Computational \& Graphical Statistics, vol. 27, no. 2, pp. 278-290, 2017.

[2] F. X. Yu, A. Bhaskara, S. Kumar, Y. Gong, and S. F. Chang, "On binary embedding using circulant matrices," Journal of Machine Learning Research, vol. 18, 2018.

[3] X. Yang, X. Tao, Y. J. Guo, X. Huang, and Q. Cui, "Subsampled circulant matrix based analogue compressed sensing," Electronics Letters, vol. 48, no. 13, pp. 767-768, 2012.

[4] H. Han, C. Tang, Y. Lou, and M. Xu, "Construction of efficient MDS matrices based on block circulant matrices for lightweight Application," Fundamenta Informaticae, vol. 145, no. 2, pp. 111-124, 2016.

[5] E. Carrasquinha, C. Amado, A. M. Pires, and L. Oliveira, "Image reconstruction based on circulant matrices," Signal Processing: Image Communication, vol. 63, pp. 72-80, 2018.

[6] D. M. Nguyen and S. Kim, "Minimal-entanglement entanglement-assisted quantum error correction codes from modified circulant matrices," Symmetry, vol. 9, no. 7, pp. 122-131, 2017.

[7] N. Y. Yu, "Indistinguishability of compressed encryption with circulant matrices for wireless security," IEEE Signal Processing Letters, vol. 24, no. 2, pp. 181-185, 2017.

[8] H. Myšková and J. Plavka, "AE and EA robustness of interval circulant matrices in max-min algebra," Fuzzy Sets and Systems, vol. 384, pp. 91-104, 2020.

[9] P. De Poi, E. Mezzetti, M. Michałek, R. M. Miró-Roig, and E. Nevo, "Circulant matrices and Galois-Togliatti systems," Journal of Pure and Applied Algebra, vol. 224, no. 11, Article ID 106404, 2020.

[10] S. Dong, P. Zhao, X. Lin, and D. Kaeli, "Exploring GPU acceleration of deep neural networks using block circulant matrices," Parallel Computing, vol. 100, Article ID 102701, 2020.

[11] S. Shen and J. Cen, "On the bounds for the norms of r-circulant matrices with the Fibonacci and Lucas numbers," Applied Mathematics and Computation, vol. 216, no. 10, pp. 2891-2897, 2010.

[12] S.-Q. Shen, J.-M. Cen, and Y. Hao, "On the determinants and inverses of circulant matrices with Fibonacci and Lucas numbers," Applied Mathematics and Computation, vol. 217, no. 23, pp. 9790-9797, 2011.

[13] D. Bozkurt and T.-Y. Tam, "Determinants and inverses of circulant matrices with Jacobsthal and Jacobsthal-Lucas
Numbers," Applied Mathematics and Computation, vol. 219, no. 2, pp. 544-551, 2012.

[14] D. Bozkurt and T.-Y. Tam, "Determinants and inverses of $r$ circulant matrices associated with a number sequence," Linear and Multilinear Algebra, vol. 63, no. 10, pp. 2079-2088, 2014.

[15] Y. Yazlik and N. Taskara, "On the norms of an $r$-circulant matrix with the generalized k-Horadam numbers," Journal of Inequalities and Applications, vol. 394, 2013.

[16] Y. Zheng and S. Shon, "Exact determinants and inverses of generalized Lucas skew circulant type matrices," Applied Mathematics and Computation, vol. 270, pp. 105-113, 2015.

[17] A. Carmona, A. M. Encinas, S. Gago, M. J. Jiménez, and M. Mitjana, "The inverses of some circulant matrices," Applied Mathematics and Computation, vol. 270, pp. 785-793, 2015.

[18] X. Jiang and K. Hong, "Explicit form of determinants and inverse matrices of Tribonacci r-circulant type matrices," Journal of Mathematical Chemistry, vol. 56, no. 4, pp. 12341249, 2018.

[19] C. Y. He, J. M. Ma, K. P. Zhang, and Z. H. Wang, "The upper bound estimation on the spectral norm of $\mathrm{r}$-circulant matrices with the Fibonacci and Lucas numbers," Journal of Inequalities and Applications, vol. 72, 2015.

[20] R. Türkmen and H. Gökbas, "On the spectral norm of r-circulant matrices with the Pell and Pell-Lucas numbers," Journal of Inequalities and Applications, vol. 65, 2018.

[21] Z. Chen, "On nonsingularity of circulant matrices," Linear Algebra and its Applications, vol. 612, pp. 162-176, 2021.

[22] B. Radičić, "On k-circulant matrices (with geometric sequence)," Quaestiones Mathematicae, vol. 39, no. 1, pp. 135144, 2016.

[23] B. Radičić, "On k-circulant matrices involving geometric sequence," Hacettepe Journal of Mathematics and Statistics, vol. 48, no. 3, pp. 805-817, 2019. 